https://doi.org/10.21670/ref.2017.37.a07

Artículos

\title{
Los efectos del DACA en la carrera profesional y las emociones de jóvenes migrantes
}

\section{The effects of DACA in young migrant's professional careers and emotions}

Magdalena Barros Nock a* (iD https://orcid.org/0000-0002-6847-8700
Recibido el 27 de marzo de 2017. Aceptado el 5 de septiembre de 2017.

*Autor para correspondencia: Magdalena Barros Nock, correo electrónico: mbarros55@hotmail. $\mathrm{mx}$

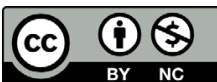

Esta obra está protegida bajo una Licencia Creative Commons Atribución-NoComercial 4.0 Internacional.
${ }^{a}$ Centro de Investigaciones y Estudios Superiores en Antropología Social, Ciudad de México, México, correo electrónico: mbarros55@hotmail.mx

\section{Resumen}

El martes 5 de septiembre de 2017 el Presidente Trump, anunció el fin del programa Acción Diferida para la Deportación de Menores (DACA) y le dio al Congreso seis meses para encontrar una salida para la vida de estos jóvenes en E.U.A. El presente artículo tiene como objetivo estudiar los efectos que el DACA ha tenido en las carreras profesionales y en las emociones de los jóvenes migrantes, a través de cuatro estancias de trabajo de campo entre 2012 y 2016 en el Valle Agrícola de Santa María, California, donde se llevaron a cabo entrevistas abiertas a jóvenes, siguiendo las transformaciones de sus estudios, trabajos y el desarrollo de sus emociones. Los datos obtenidos son cualitativos. En el estudio vimos como los jóvenes se esforzaron por continuar sus estudios y buscar empleos fuera del sector agrícola. Sus emociones pasaron del miedo y la desesperanza a sentimientos de esperanza. Sin embargo, una vez más el miedo regresó a sus vidas.

Palabras clave: DACA, jóvenes migrantes, carrera profesional y emociones, California, Estados Unidos.

\section{Abstract}

Tuesday 5 of September of 2017 President Trump announced the end of Deferred Action for Childhood Arrivals (DACA) and gave Congress six months to find a solution for the lives of these young people in the us. The present article has the objective to study the effects that DACA has had on the professional careers of young migrants and their emotions through four field work stays between 2012 and 2016 in the agricultural valley of Santa Maria, California, open interviews were implemented to young women and men, following the transformations in their studies, work and emotions. The data obtained is qualitative. In the study, we can see how the young made an effort to continue their studies and search for employment outside the rural sector. Their emotions went

CÓMO CITAR: Barros, M. (2017). Los efectos del DACA en la carrera profesional y las emociones de jóvenes migrantes [The effects of DACA in young migrant's professional careers and emotions]. Estudios Fronterizos, 18(37), 131-148, doi:10.21670/ref.2017.37. a07 
from fear and lack of hope to feelings of hope. However, fear has come back again.

Keywords: DACA, young migrants, professional career and emotions, California, United States.

\section{Introducción}

El presente artículo trata sobre la vida de los jóvenes que arribaron a Estados Unidos de niños con sus familias y crecieron en ese país, estudiaron en sus escuelas, muchos de ellos terminaron la preparatoria, algunos lograron entrar al colegio estatal, otros a la universidad. Crecieron pensando que pasarían el resto de su vida en Estados Unidos, pues sus sueños y expectativas estaban hechas allá; sin embargo, todos en distintos momentos enfrentaron la crudeza que significa vivir sin documentos. Algunos desde pequeños vivieron la experiencia de la deportación de uno de sus padres, la profunda tristeza de su ausencia y el miedo constante de perder al otro; otros enfrentaron día a día el bullying en la escuela donde se les señala y estigmatiza por ser indocumentados. Pero el enfrentamiento abierto empieza al finalizar la preparatoria e intentar entrar al colegio estatal o la universidad y ver su camino constantemente bloqueado, con obstáculos y retos que sus amigos e incluso hermanos con ciudadanía no enfrentan; o al tratar de buscar un buen trabajo y no poder encontrarlo por no tener papeles que los avalen como residentes o ciudadanos.

Gracias a la organización y presión de miles de jóvenes llamados dreamers $^{1}$ el presidente Barack Obama decidió implementar en 2012 una acción ejecutiva presidencial llamada DACA² (Acción Diferida de la Deportación para los Menores), que les permitió obtener un permiso para estudiar y trabajar, sin embargo, este programa no solucionó de manera definitiva el problema de estos jóvenes, solo dio un alivio temporal, al ser un permiso de dos años, renovable y sujeto a la decisión presidencial de ser revocado en cualquier momento, tal como sucedió el martes 5 de septiembre de 2017 Jeff Sessions, Fiscal General de los Estados Unidos, anunció a nombre del presidente Trump que daba por terminado el programa DACA.

El actual presidente de Estados Unidos Donald Trump, durante su campaña expresó su intención de terminar con todas las acciones ejecutivas llevadas a cabo por el presidente Obama. En conferencia de prensa dada el 16 de febrero de 2017, dijo que tendría una solución para los jóvenes DACA, pero que incluso entre estos jóvenes había miembros de pandillas y por lo tanto había que aplicarles la ley. Dos días antes, el 14 de febrero, ya un joven DACA había sido apresado por el ICE$^{3}$ para ser deportado (Levine y Cooke, 2017). El presidente Trump se había mantenido ambivalente por varios meses frente al programa DACA, pero 10 jueces de varios estados amenazaron con demandarlo si para el 5 de septiembre no terminaba con DACA (Dinan, 2017),

\footnotetext{
${ }^{1}$ Este nombre proviene del Dream Act (Development Relief and Education for Allien Minors) propuesto al senado por dos senadores en 2001 y que no fue aceptado.

${ }^{2}$ Por sus siglas en inglés Deferred Action for Childhood Arrivals.

${ }^{3}$ ICE en inglés significa U.S. Immigration and Customs Enforcement. El ICE pertenece al Departamento de Seguridad Nacional (US Department of Homeland Securtity) y tiene como objetivo identificar e investigar cualquier problema concerniente a la seguridad de la frontera de Estados Unidos. Parte de su trabajo son las llamadas remociones o lo que en México llamamos deportaciones.
} 
así que Trump decidió cumplir con su promesa de campaña y dio por terminado este programa. Su propuesta le da seis meses al Congreso para que encuentre una solución para los jóvenes DACA, pero tenemos que recordar que Obama se vio en la necesidad de utilizar una Acción Ejecutiva Presidencial porque el Congreso no estaba dispuesto a llevar a cabo ningún tipo de medida que ayudara a los migrantes indocumentados. ${ }^{4}$ Esta decisión pone a alrededor de 800000 jóvenes y sus familias de nuevo en una situación de miedo y angustia, con un futuro incierto.

En este escenario, el futuro de los jóvenes DACA queda en manos del Congreso y de la gente que los apoya; los siguientes meses serán de lucha en las calles, en los teléfonos, con cartas de apoyo dirigidas a los congresistas, es necesario utilizar todas las herramientas existentes para apoyar a estos jóvenes y lograr movilizar a los legisladores. Mientras tanto, hay miedo e inseguridad entre los jóvenes y esta incertidumbre está teniendo un efecto negativo en ellos y sus familias.

Es importante exponer cómo el DACA ha marcado a estos hombres y mujeres jóvenes, por lo que este estudio presenta los cambios ocurridos en la vida de los beneficiarios del DACA entre los años del 2012 al 2016, tanto a nivel profesional como en sus emociones, con el objetivo de entender el impacto que este programa tuvo en sus vidas y cotidianidad.

Para finales del 2016 había poco más de 752000 jóvenes en el programa DACA (Mathema, 2017). El estado con el mayor número de jóvenes DACA es California con 216 060, le sigue Texas con 120642 y Carolina del Norte con 26 936. Se calcula que $80 \%$ de ellos se encuentran trabajando y el resto estudiando (Mathema, 2017). En los últimos meses en la zona de estudio, el Valle Agrícola de Santa María, California, la renovación de los permisos DACA casi se detuvo. Al momento de las elecciones las organizaciones activistas recomendaron que nadie realizara las gestiones de registro hasta saber cómo reaccionaría el presidente Trump, quien por meses manifestó la intención de terminar con el DACA en cuanto entrara a la Casa Blanca. Ahora las amenazas se han cumplido.

Estos jóvenes se enfrentan a un Estado cada vez más duro en sus políticas hacia los migrantes indocumentados que por décadas los ha criminalizado, llevando hasta las últimas consecuencias esta forma de regular la migración, de manera que la vida de los migrantes indocumentados y sus familias ha sido cada vez más difícil, precaria y violenta (De Genova, 2002; Dowling e Inda, 2013). Esta situación se agravó durante la campaña presidencial, en la cual se creó un "efecto Trump" donde el entonces candidato abiertamente insultó y mostró actitudes racistas hacia la población mexicana, propiciando de esta manera que otros siguieran su ejemplo (Barros, 2017). Aún ocho meses después de tomar posesión de su cargo, Trump continuó mostrando su cara racista, un ejemplo reciente de ello fue la posición poco firme en contra de los grupos de supremacía blanca que se manifestaron en Charlottesville, Virginia el sábado 12 de agosto, donde murió una mujer (Merica, 2017). Esta ambivalencia frente a actos y grupos racistas afecta a todas las comunidades de migrantes, ya que los sentimientos xenofóbicos contra ellos emergen con mayor frecuencia y fuerza.

\footnotetext{
${ }^{4}$ Puntos clave: a) ya no se aceptarán nuevas solicitudes para ingresar al DACA; $b$ ) se aceptarán renovaciones hasta el 5 de octubre de 2017; c) para los casos en que el DACA expire para el 5 de marzo de 2018, se señala que deben renovar antes del 5 de octubre de 2017 y d) que a partir de estas fechas una vez que se terminen los permisos, los muchachos vuelven a ser indocumentados.
} 
Los jóvenes DACA llegaron de niños o adolescentes a Estados Unidos y se han integrado al sistema educativo americano, algunos con más éxito que otros, logrando articularse al sistema escolar y llegar a niveles del colegio estatal e incluso a la universidad; sin embargo su camino no ha sido fácil, muchos se han encontrado con múltiples obstáculos en su tránsito por la preparatoria que los ha llevado a no recibir su diploma o no poder aspirar a los cursos que querían por ser indocumentados. Estos niños se están convirtiendo en adultos y su futuro, en mucho, depende de su situación legal en Estados Unidos.

En este documento presentaremos algunos de los efectos que el DACA ha tenido en la vida de jóvenes que viven en el valle agrícola de Santa María —localizado en la costa central de California- y que son miembros de familias de estatus legal mixto, esto significa que pertenecen a familias donde algunos de sus miembros pueden tener la ciudadanía, otros solo la residencia o permiso para trabajar y otros son indocumentados. La mayoría de sus padres llegaron al valle para trabajar en actividades agropecuarias, ya sea como jornaleros o en las congeladoras y semilleros. Algunos han podido dejar el trabajo en el campo y conseguir empleo en la construcción, sobre todo en los tiempos en que esta actividad tuvo su auge, así como en los servicios y comercio, aunque la gran mayoría trabaja en el campo (Barros, 2015a).

Este artículo está basado en información recabada durante cuatro visitas de trabajo de campo de seis semanas cada una, entre los años 2012 y 2016 . Durante estos años tuve la oportunidad de entrevistar a 11 jóvenes entre 18 y 30 años sobre el DACA y el efecto que estaba teniendo en sus vidas. De estos, cinco son mujeres y seis hombres que tenían entre 4 y 11 años de edad cuando llegaron a los Estados Unidos con sus familias.Todos son mestizos y provienen de diferentes partes de México. Y metieron sus solicitudes al DACA y fueron acepados. Además de ellos entrevisté a otros 25 jóvenes, de los cuales 25 por ciento no metieron sus papeles al DACA, ya sea porque no calificaron para hacerlo o porque decidieron no hacerlo aún. Tres estaban casados y tenían hijos. Los entrevisté en varias ocasiones, una vez cuando consiguieron su permiso y una o dos veces más a lo largo de los cuatro años, entre 2013 y 2016. Utilicé el método bola de nieve para localizar a los entrevistados, uno me llevaba a otro. También asistí a organizaciones que los estaban ayudando a llenar sus solicitudes y ellos les preguntaban si querían entrevistarse conmigo; de aceptar, arreglaban una entrevista.

Las entrevistas fueron abiertas, entre las preguntas se les pedía indicar si sus vidas habían cambiado desde que entraron al programa DACA y qué características tenían estos cambios. En las respuestas los jóvenes mencionaban principalmente dos aspectos en los que su vida estaba siendo afectada desde que entraron al programa: el primero es lo que en este artículo llamaremos su carrera profesional, que incluye todas las actividades que pudieron desarrollar tanto educativas como laborales, y que han venido a mejorar su nivel económico actual y sus expectativas para el futuro. Como segundo aspecto, hacían referencia a sus emociones, los jóvenes describían de qué manera el miedo que antes sentían de ser descubiertos como indocumentados y ser deportados a un país que no conocían había desaparecido una vez que tenían su permiso; esto traía cambios en otras emociones y en las relaciones con su familia, sus amigos, la comunidad y en la percepción de su futuro en EE. uU. Sin embargo, conforme la campaña de Trump empezó a adquirir fuerza y ganó la presidencia, la inseguridad y el temor regresaron a sus vidas. 
Este artículo se divide en dos secciones, en la primera se describen los efectos que el DACA ha tenido en las carreras profesionales de los jóvenes, se resalta el interés que ha emergido entre ellos por seguir estudiando para mejorar sus oportunidades laborales en el futuro próximo y en las posibilidades existentes de conseguir mejores trabajos una vez que han regularizado su situación legal. En la segunda parte se analizan aspectos subjetivos, en específico cómo el DACA ha influido en las emociones de estos hombres y mujeres jóvenes, concentrándonos en las emociones de miedo, decepción, desesperanza y esperanza, y se pone atención en los efectos que el DACA ha tenido de manera diferenciada entre mujeres y hombres. ${ }^{5}$

En las últimas décadas la violencia contra los migrantes indocumentados ha aumentado. La mayoría de los jóvenes entrevistados llegaron siendo pequeños a Estados Unidos y en un principio no sabían que eran indocumentados hasta que llegaron a ser adolescentes e incluso algunos hasta el último año de la High School (preparatoria) cuando tuvieron que tomar decisiones sobre su futuro y vieron que sus alternativas estaban limitadas debido a su condición de migrantes sin documentos; sin embargo, siendo miembros de familias de estatus legal mixto, desde pequeños ya sentían la presión y el miedo que los miembros de su familia que no tenían documentos vivían día a día, algunos incluso han experimentado la deportación de sus seres queridos. Se han visto expuestos a los procedimientos disciplinarios que el gobierno ejerce sobre los migrantes indocumentados tales como las tecnologías biométricas, la criminalización a partir del marco legal, la restricción en el uso de los servicios públicos, además del miedo a ser detenidos en cualquier momento, incluso en su propia casa al amanecer, el posible encarcelamiento y su consecuente deportación, el miedo constante a ser en cualquier momento deportados (Aquino, 2015; Barros, 2017).

\section{El contexto: El valle agrícola de Santa María, el movimiento dreamer y DACA}

Desde principios del siglo pasado un flujo constante de migrantes ha llegado al valle de Santa María. En 1942 se inició el Programa Bracero y con él camiones llenos de trabajadores cada año al inicio del ciclo agrícola arribaban al valle, así como trabajadores indocumentados en busca de trabajo y, vivían en los campamentos para jornaleros agrícolas ubicados en los campos.

En los años cincuenta el valle experimentó un auge agroindustrial importante, se construyeron agroindustrias, incluyendo invernaderos de flores y vegetales, plantas fertilizadoras, constructoras de cajas, empacadoras, etc. Algunos braceros y exbraceros comenzaron a vivir en las orillas de los pueblos rentando cuartos; algunos mexicanos para entonces lograron establecer algunos negocios para servir a la creciente población mexicana (Barros, 2009).

En la década de los años sesenta, el fin del Programa Bracero en 1964 marcó el inicio del asentamiento de mexicanos en forma constante y creciente. La discriminación

\footnotetext{
${ }^{5}$ Para un análisis sobre cómo los jóvenes migrantes indígenas mixtecos han percibido y reaccionado frente al DACA ver Barros y García (2015).
} 
hacia los mexicanos era muy fuerte y solo pocas familias se habían aventurado a abrir negocios o comprar una casa. No obstante, cambios importantes estaban sucediendo entre la población mexicana, es de mencionarse el movimiento de César Chávez que tendría un impacto muy importante en la vida económica y política de los jornaleros agrícolas y en la vida de los mexicanos inmigrantes en general. Esto sería el inicio de la toma de conciencia de las posibilidades y derechos del trabajador agrícola, como empleado y como ciudadano en Estados Unidos, lo cual se reflejaría en su interés por su ciudad.

La principal actividad económica del valle era y sigue siendo la agricultura y su agroindustria. Para finales de los años setenta y principios de los ochenta la agricultura sufrió una serie de cambios, la producción de frutas y verduras se introdujo de forma masiva en el valle, lo cual demandó más mano de obra, además el incremento en el uso de tecnología en los cultivos alargó las cosechas y los salarios se elevaron. Este aumento de oferta de trabajo durante el año dio la posibilidad al trabajador agrícola de permanecer por periodos más prolongados y establecerse en Estados Unidos, lo que propició que la población de origen mexicano de diversos estados de México fuera atraída a California por la oferta de trabajo en el campo y la agroindustria, así como por los altos salarios.

La amnistía en 1986 (Immigration Reform and Control Act [IRCA]) les dio la posibilidad a muchos inmigrantes de obtener su residencia y eventualmente la ciudadanía. Con el programa de reunificación familiar muchas familias volvieron a unirse. Pero en tiempos recientes la creciente militarización de la frontera ha hecho cada vez más difícil, peligroso y costoso el ir y venir de los trabajadores, lo cual los lleva a quedarse por periodos cada vez más largos.

Estos trabajadores provenientes de diferentes estados de México, especialmente de Jalisco, Irapuato, Zacatecas y Oaxaca, se encuentran insertos en una de las regiones agrícolas más productivas del mundo; se han ido incorporando poco a poco, a través de las décadas, a esta agricultura capitalista. Aun cuando el trabajo en el campo es un trabajo mal pagado, que se lleva a cabo bajo condiciones extenuantes, entre insecticidas y plaguicidas, que afecta y deteriora su salud.

En estas condiciones migraron con sus hijos pequeños, asentándose en el valle. Muchas de estas familias también tuvieron hijos en Estados Unidos. De esta manera los hijos mayores llegaron cuando eran pequeños y crecieron en Estados Unidos sintiéndose parte de la sociedad americana, pero sin documentos, mientras que los hijos menores que nacieron en Estados Unidos tienen la ciudadanía americana.

La crisis económica empezó a sentirse desde mediados de la década del 2000. En 2008 se declaró oficialmente el inicio de la recesión en Estados Unidos, sin embargo, esta forma parte de una crisis global que afectó al mundo entero. Uno de los grupos más afectados, incluso antes de que oficialmente se declarara la recesión, fueron los latinos. De 2007 a 2008 el número de desempleados hispanos nacidos fuera de Estados Unidos subió de $5.1 \%$ a $8.0 \%$ a nivel nacional, y el de hispanos nacidos en Estados Unidos fue de $6.7 \%$ a $9.5 \%$. El de la población general fue de $4.6 \%$ a $6.6 \%$, como vemos mucho menor (Kochhar, 2009).

El empleo en el sector de la construcción y todos los negocios que lo abastecen venían ya en picada desde 2007, y era uno de los sectores que más trabajadores latinos contrataba: $54.2 \%$ de los empleos perdidos por los latinos se encontraban en el sector de la construcción (Kochhar, 2008). Para 2008 la producción económica había caído a niveles más bajos que en 1930 (Cornelius, FitzGerald, Lewin y Muse- 
Orlinoff, 2010), y los latinos estaban perdiendo sus empleos más rápido que cualquier otro grupo en los Estados Unidos (Kochhar, 2009). Para 2010, la población total de California era de 37253 956, de los cuales $30.7 \%$ era de origen mexicano. Para esas fechas, California contaba con una tasa de 12\% de desempleo, de los cuales, $14 \%$ eran hispanos (United States Census Bureau, 2010). En 2010 la ciudad de Santa María tenía una población de 99553 habitantes, de los cuales 70114 eran de origen mexicano, es decir, $70.42 \%$, con un desempleo de 5.2\% (United States Census Bureau, 2010).

Mientras Estados Unidos enfrentaba estos problemas, había una generación de jóvenes que estaba creciendo en las escuelas y luchando por entrar a los colegios estatales y a las universidades. Una generación que luchó por la educación y llegaron a conocer estrategias para mejorar su situación y la de sus familias en E.u. Estos espacios les permitieron conocer sus derechos y las posibilidades de lucha que tenían para cambiar su situación de indocumentados. Algunos pasaron de ser indocumentados a activistas y decidieron que la mejor estrategia era dar a conocer su situación. Contactaron a senadores y representantes para dar visibilidad a miles de jóvenes como ellos. Así surgió el movimiento dreamer, el cual motiva a los jóvenes a luchar contra la criminalización de los migrantes y a hacerse visibles ante la sociedad y el gobierno (Truax, 2013). El movimiento dreamer tuvo un impacto diverso en las zonas rurales. En el caso de Santa María existe un pequeño grupo de dreamers formado por 10 estudiantes en la Allan Hanckok College que llevan a cabo diversas actividades para recaudar dinero que usan para ayudar a los alumnos indocumentados a pagar sus inscripciones y sus libros, sin embargo, fuera de este círculo, son pocos los jóvenes que para el año 2016 conocían el movimiento y menos aún que participaran activamente en él.

El movimiento dreamer ha obtenido beneficios para los jóvenes indocumentados en Estados Unidos, uno de los más importantes fue atraer la atención del gobierno de Obama y del público en general a la situación de esta generación que llegaron cuando eran niños y niñas con sus familias y crecieron en Estados Unidos, llamados generación 1.5. Después de más de dos décadas de no existir ninguna amnistía o medida de apoyo a los migrantes para que pudieran obtener su residencia, el presidente Obama y su administración pusieron en marcha en junio de 2012 el DACA, que es una medida para proteger temporalmente de ser deportados a los jóvenes que llegaron de niños a Estados Unidos, tiene vigencia de dos años con la posibilidad de ser renovado; no obstante, no es un permiso de residencia que pueda llevar eventualmente a la ciudadanía.

La acción diferida para la deportación otorga a jóvenes que reúnen ciertas condiciones la posibilidad de solicitar permisos para trabajar por dos años, seguro social y licencia en algunos estados como California no obstante, el DACA podía ser renovado o revocado por Donald Trump. Los requisitos impuestos por esta acción ejecutiva a su vez han dejado fuera a muchos jóvenes que no califican y que por diversas razones han preferido no "meter sus papeles", entre estas razones está la falta de dinero, la falta de confianza en el sistema y el miedo a que una vez que tengan sus datos los quieran deportar junto con sus familias; o bien el creer que es injusto que dejen fuera a sus padres de un programa que da alivio a una situación cada vez más difícil para toda la familia (Barros, 2015b). 
Gracias a la lucha de los miembros del movimiento United We Dream que dio origen a la propuesta Dream Act, ${ }^{6}$ en una acción que muchos consideran un movimiento con fines electorales, el presidente Obama presentó la Iniciativa "temporal" de Acción Diferida para los llegados en la Infancia (DACA) que entró en vigor el 15 de agosto de 2012 donde se esperaba que más de 1.4 millones de jóvenes con características específicas $^{7}$ pudieran incorporarse al programa y recibir un retardamiento a su deportación y permiso para trabajar por dos años. Esta medida está dirigida a jóvenes que fueron llevados como niños a los Estados Unidos. Se calcula que de esta cifra aproximadamente 1.1 millones son de origen mexicano, es decir $65 \%, 60000$ provienen de El Salvador y 50000 de Guatemala, y $57 \%$ de los potenciales beneficiados viven en California, Texas, Florida, Nueva York e Illinois ("Beneficiarán a más 'Soñadores'”, 2012).

Por otro lado, el presidente Obama ha sido uno de los presidentes con el mayor número de deportaciones en la historia contemporánea de Estados Unidos, acción que ha separado familias y dejado a niños sin sus padres, creando un ambiente de miedo y marginación donde los niños y jóvenes temen que en cualquier momento sus padres o ellos mismos en el caso de ser indocumentados, puedan ser arrestados y deportados por las autoridades americanas. ${ }^{8}$

No cabe duda que DACA fue un gran paso en la ayuda a miles de jóvenes indocumentados que llegaron de niños a Estados Unidos y que viven dentro de las familias de estatus legal mixto. Las crecientes deportaciones han afectado especialmente a estas familias. Para 1998, 27\% de las familias con hijos en California eran familias de estatus legal mixto (Fix y Zimmermann, 2001, p. 399). En estas familias encontramos hijos que nacieron en Estados Unidos y que son ciudadanos por nacimiento, e hijos que fueron traídos de pequeños y que han permanecido como indocumentados. La posibilidad de que ellos o sus padres sean deportados en cualquier momento ha hecho que sus vidas se desenvuelvan en un ambiente no solo de marginación y discriminación sino también de miedo y creciente violencia.

\footnotetext{
${ }^{6}$ Dream es el acrónimo de Development, Relief and Education for Alien Minor. Básicamente lo que piden los miembros que promueven el Dream Act es que los jóvenes indocumentados que quieran ingresar a la universidad reciban las mismas condiciones que los jóvenes residentes, y no se hagan diferencias basadas en origen étnico y nacional. Los jóvenes indocumentados llegan a pagar colegiaturas en las universidades hasta tres veces más altas que los residentes, lo cual dificulta mucho su integración. Esta iniciativa fue presentada al congreso en septiembre 2006 y no ha sido aprobada. El único estado que aprobó su propio Dream Act fue California en 2013.

${ }^{7}$ Los jóvenes necesitan tener las siguientes características: tener menos de 31 años a la fecha de junio de 2012; haber llegado a los Estados Unidos antes de los 16 años de edad; haber residido continuamente en los Estados Unidos por un periodo mínimo de tres años antes del 15 de junio de 2012 y en el momento de presentar la acción diferida ante la United States Citizenship and Immigration Services (USCIS); haber residido continuamente en los E.U. desde junio 15 de 2007 hasta el momento presente; estar asistiendo a la escuela, o haberse graduado de la escuela superior, poseer un certificado de Educación General (GED por sus siglas en inglés), o haber servido con honor en la Guardia Costera o en las Fuerzas Armadas de los Estados Unidos. No haber sido encontrado culpable de un delito grave, delito menos grave significativo, múltiples delitos menos graves, o representar amenaza nacional o a la seguridad pública (USCIS, 2012).

${ }^{8}$ Solo en los primeros seis meses de 2012 se deportaron 45000 padres y madres y más de 5100 niños ciudadanos en 22 estados viven bajo el cuidado del Estado en los programas llamados foster care donde familias cuidan a los niños y niñas temporalmente hasta que alguna familia los adopte definitivamente 0 lleguen a la edad adulta y se pueden cuidar por sí mismos (O’Neil, 2012).
} 
En 2012 se aceptaron 152423 solicitudes y 5395 fueron rechazadas; en $2013,{ }^{9}$ 427601 fueron aceptadas y 16356 rechazadas; para 2014, 122444 fueron aceptadas y 19136 rechazadas. En 2014 empezó la renovación de los primeros jóvenes que sacaron su DACA, en ese año 116441 renovaron su DACA y 5762 fueron rechazadas. En 2015, 26696 jóvenes solicitaron DACA por primera vez, 2287 fueron rechazados, 118550 han renovado, y 6886 fueron rechazados. Hasta el 2015, un total de 727164 solicitudes habían sido aceptadas y 43174 rechazadas, de las cuales 234991 ya llevan una renovación (USCIS, 2015).

\section{El efecto del DACA en la carrera profesional de los jóvenes}

Para analizar de qué forma el DACA ha afectado la vida laboral de los jóvenes nos basaremos en el concepto de carrera que fue originalmente desarrollado por Hughes (1960). Este fue utilizado como una herramienta analítica para estudiar la secuencia de movimientos de una posición a otra en el sistema ocupacional, hechos por un individuo que trabaja dentro de un sistema. Hughes desarrolló un modelo que toma en cuenta factores externos objetivos como son la estructura de oportunidades y las limitantes existentes, así como factores subjetivos tales como cambios en la percepción, motivación y deseos de cada individuo (Hughes, 1960).

En el apartado anterior presenté el contexto en que los jóvenes crecieron que nos da una idea de la estructura de oportunidades que existen en la ciudad en que viven, inmersa en un valle agrícola donde la actividad principal es la agricultura y la agroindustria que la apoya. Es importante mencionar este contexto ya que los padres de los jóvenes entrevistados, con excepción de dos, trabajan ya sea como jornaleros en el campo o en la agroindustria. Hablando con algunos de sus padres, todos expresaron haber venido a Estados Unidos con el sueño de que sus hijos e hijas pudieran tener una mejor vida que la que ellos tenían, todos querían que sus hijos recibieran una educación y pudieran conseguir trabajos fuera de la agricultura. Los jóvenes a su vez expresaban el mismo deseo de continuar sus estudios para poder conseguir trabajos fuera de la agricultura, con mejores condiciones laborales. Carmen comenta:

Cuando terminé la High School solo encontré trabajo en el campo, un trabajo duro, cansado, so, yo quería estudiar y hacer otra cosa, algo fuera del sol, de los insecticidas. Yo he visto como mis padres sufren en el campo, yo no quiero eso you know (Carmen, Santa María, 2015).

Los jóvenes saben lo difícil de la situación laboral de sus padres y ellos quieren algo distinto en sus vidas. Veremos primero cuál era su situación escolar antes del DACA y si DACA les ha permitido continuar o terminar sus estudios y posteriormente hablaremos sobre los trabajos que han obtenido antes.

\footnotetext{
${ }^{9}$ Para agosto de 2013 solo 49\% de la población elegible, según cifras del gobierno de Obama, había presentado su solicitud; de estos $68 \%$ provenían de México, El Salvador, Guatemala y Honduras; $24 \%$ se encontraban estudiando la preparatoria y $44 \%$ no estaban inscritos en la universidad ni tenían un título universitario (Wong et al., 2013).
} 
Estos jóvenes entraron a la escuela cuando llegaron a Estados Unidos, pero no todos terminaron la preparatoria. ${ }^{10}$ De los jóvenes entrevistados solo $70 \%$ terminó su preparatoria.

Alfredo nos dice:

Yo llegué a los 14, mi papá me metió a la escuela porque aquí es obligatorio, pero él quería que yo trabajara en el campo con él. Necesitaba mi ayuda. En la escuela me costó trabajo aprender inglés y no entendía lo que estaban haciendo. No obtuve mi diploma. Pero cuando supe del DACA me metí a clases y estoy por sacarlo. Yo quiero salirme del campo y el DACA es mi oportunidad (Alfredo, Santa María, 2013).

Como Alfredo, muchos de los que no habían podido obtener su diploma de la High School, cuando escucharon sobre el DACA, se metieron a las clases que se ofrecían en el colegio estatal y otros centros autorizados y sacaron su certificado de estudios. ${ }^{11}$

Sin embargo, aun cuando terminaran la preparatoria, la mayoría, se enfrentó con una serie de obstáculos por ser indocumentados para poder seguir estudiando, siendo los principales el tener que pagar cuotas de inscripción altas, libros caros, no poder competir por los mismos apoyos financieros que jóvenes con status legal. Algunos lo han logrado sin documentos. Felicia comentaba:

En el college no me dejaban tomar el curso de cómputo pues este para indocumentados costaba más caro, so, solo para aquellos que tenían papeles. Solo cuando obtuve mi DACA pude tomar el curso. (Felicia, Santa María, 2015).

Estos jóvenes perdían la esperanza de continuar con la carrera profesional que ellos habían pensado podrían hacer en su adolescencia. Por ejemplo, Felipe nos dice:

Yo quería estudiar para ser enfermero, you know, siempre me ha gustado la medicina y ayudar a la gente. So, sin documentos no valía la pena ya que en ningún lugar me contratarían. So, no continué mis estudios you know y entré a trabajar de mesero. Cuando saqué mi DACA luego luego entré a estudiar y ahora estoy haciendo mi internado. Soy muy feliz, I am doing my dreams, finally, thank you Obama!! (Felipe, Santa María, 2012).

Felipe logró salir de empleos con salarios bajos, que no lo conducían a realizar su sueño, hacer lo que a él más le atraía, ayudar a la gente, estudiar para ser enfermero. Este chico, sin el DACA, no hubiera podido realizar esta profesión que tiene una alta demanda en estos momentos en Estados Unidos. Al igual que en el caso de Felipe, se entrevistó a otros dos jóvenes DACA que están estudiando enfermería, que es una carrera donde hay oportunidades de trabajo en la región con la que aspiran a tener un buen salario.

\footnotetext{
${ }^{10}$ Es importante explicar que hay una gran diversidad en cuanto a la edad que estos jóvenes tenían cuando llegaron a Estados Unidos. Algunos llegaron de niños y entraron a la primaria. Para ellos era más fácil aprender el inglés. Conforme son mayores, les fue más difícil aprender el idioma y adaptarse al sistema de estudios y tienen más probabilidades de no recibir su diploma de la High School. De los jóvenes entrevistados, cuatro mujeres entraron a la primaria cuando llegaron y una a la secundaria, dos hombres a la primaria y cuatro a la secundaria. Ninguno llegó en edad de entrar a la preparatoria. Dominar el idioma inglés, conocer el sistema educativo americano y tener un diploma de High School le da ventajas a los jóvenes que quieren aplicar al DACA. Este es un tema que necesita más investigación. Para profundizar en el tema de la educación y el lenguaje ver Suárez-Orozco, Suárez-Orozco y Todorova (2008).

${ }^{11}$ Hay que tomar en cuenta que durante la presidencia de Obama, los niños y niñas que crecían y llegaban a cumplir con la edad que se requería para aplicar a DACA, realizaban su aplicación.
} 
La Tabla 1 muestra los cambios que los jóvenes entrevistados han tenido en sus carreras, podemos ver como ninguno había terminado sus estudios en el colegio estatal e incluso uno no había terminado su preparatoria. Ahora todos están estudiando cosas que les atraen y con las cuales podrán no solo obtener mejores trabajos y ser más productivos para la sociedad sino también vivir más felices y sentirse que realizan algo que les gusta hacer, como lo comenta arriba Felipe. Por su parte Carmen (2014) nos dice: "gracias al DACA estoy haciendo algo que me gusta, I am happy, you know". La mayoría han podido encontrar a nuevos trabajos y mejor pagados a partir que tienen su DACA.

Tabla 1: Estudios y empleo antes y después de DACA (2012-2016)

\begin{tabular}{|c|c|c|c|c|}
\hline Nombre & $\begin{array}{l}\text { Estudios } \\
\text { antes de } \\
\text { DACA }\end{array}$ & $\begin{array}{l}\text { Estudios después } \\
\text { de DACA }\end{array}$ & $\begin{array}{l}\text { Trabajo antes } \\
\text { de DACA }\end{array}$ & $\begin{array}{l}\text { Trabajo des- } \\
\text { pués de DACA }\end{array}$ \\
\hline Carmen & Preparatoria & Estética & Jornalera & $\begin{array}{l}\text { En salón de } \\
\text { belleza }\end{array}$ \\
\hline Felicia & Preparatoria & $\begin{array}{l}\text { Trabajadora so- } \\
\text { cial }\end{array}$ & $\begin{array}{l}\text { Puesto } \\
\text { remate de la } \\
\text { familia }\end{array}$ & $\begin{array}{l}\text { Aún no encuen- } \\
\text { tra otro trabajo }\end{array}$ \\
\hline Cata & $\begin{array}{l}\text { Colegio } \\
\text { estatal-pero } \\
\text { no terminó }\end{array}$ & $\begin{array}{l}\text { Regresó a } \\
\text { estudiar Justicia } \\
\text { Criminal en la } \\
\text { Universidad }\end{array}$ & Agroindustria & $\begin{array}{l}\text { Sigue estu- } \\
\text { diando }\end{array}$ \\
\hline Desire & Preparatoria & $\begin{array}{l}\text { Colegio esta- } \\
\text { tal-asistente } \\
\text { médico }\end{array}$ & Jornalera & $\begin{array}{l}\text { Cuidadora de } \\
\text { mayores (sigue } \\
\text { estudiando) }\end{array}$ \\
\hline Teresa & Preparatoria & $\begin{array}{l}\text { Colegio estatal } \\
\text { Trabajadora } \\
\text { social }\end{array}$ & Jornalera & $\begin{array}{l}\text { Organización } \\
\text { no lucrativa }\end{array}$ \\
\hline Julio & $\begin{array}{l}\text { Colegio } \\
\text { estatal }\end{array}$ & $\begin{array}{l}\text { Está juntando } \\
\text { dinero para ir a la } \\
\text { universidad }\end{array}$ & $\begin{array}{l}\text { Jornalero, } \\
\text { mayordomo, } \\
\text { lavaplatos }\end{array}$ & $\begin{array}{l}\text { Vende de- } \\
\text { partamentos } \\
\text { y administra } \\
\text { departamentos }\end{array}$ \\
\hline Pedro & Preparatoria & Colegio estatal & Jornalero & Tienda \\
\hline Arturo & Preparatoria & $\begin{array}{l}\text { Estudiante de } \\
\text { arte }\end{array}$ & $\begin{array}{l}\text { Trabajaba } \\
\text { limpiando } \\
\text { oficinas }\end{array}$ & $\begin{array}{l}\text { Sigue con el } \\
\text { mismo trabajo }\end{array}$ \\
\hline Felipe & Preparatoria & Enfermería & Jornalero & $\begin{array}{l}\text { Interno en un } \\
\text { hospital }\end{array}$ \\
\hline Aurelio & Preparatoria & Ingeniería & Lava platos & Mecánico \\
\hline Alex & Preparatoria & $\begin{array}{l}\text { Terminó prepa- } \\
\text { ratoria y colegio } \\
\text { estatal }\end{array}$ & Semillero & Bombero \\
\hline
\end{tabular}

Fuente: Elaboración propia.

Como podemos apreciar el DACA les ha ayudado a desarrollar una carrera profesional que les está permitiendo crear un futuro diferente al trabajo agrícola, que 
ha mostrado no solo mantener a sus padres en la pobreza y continua precariedad, sino también golpea su salud fuertemente. El DACA da a los jóvenes permiso para trabajar, y en California antes de que el estado aprobara las licencias para todos los indocumentados en enero de 2014, los jóvenes que habían obtenido su DACA podían aplicar para obtener su licencia. Podían también seguir estudiando y conseguir apoyos financieros para sus estudios, cosa que no obtenían sin papeles.

Como opina Gerardo:

Antes del DACA yo pensé que trabajaría en el campo toda mi vida como mi papá. So, en la prepa tenía muchas ilusiones y luego me di cuenta de mi situación, fue una gran desilusión. ¡But con el DACA mi vida cambio! (Gerardo, Santa María, 2015).

\section{EI DACA y las emociones de los jóvenes}

En esta condición donde se regula la migración a través de la criminalidad y se mantiene al migrante en una situación de miedo y terror a ser deportado en cualquier momento para poder explotar su fuerza laboral y hacer de ellos lo que el capital crea conveniente, es donde estos pequeños crecen, en un principio pensando que son americanos, iguales a sus hermanos pequeños, amigos y compañeros de la escuela y poco a poco se van dando cuenta que son diferentes, que no tienen derechos, que sus futuros son distintos y el miedo empieza a entrar en sus vidas. En algunos poco a poco y en otros de un día a otro debido a eventos que los marcan, como por ejemplo la repentina deportación de alguno de sus padres, situación que los lleva a darse cuenta que su vida jamás será como habían soñado que sería.

Nos relata Ana:

Hace unos años, cuando era una niña, aún estaba dormida cuando oí que tocaban la puerta, duro, todos nos despertamos, mi mamá me metió debajo de la cama con mi hermano. Me dijo: "no salgan". Después de un largo rato, salimos, pos mi mamá llorando, se habían llevado a mi papá los de la migra. Sentí un dolor fuerte, duro en el estómago, I felt fear, no me dejó por años. ¿Pensaba... la siguiente seré yo? Esa sensación de miedo se me quitó cuando me dieron mi DaCA. Por fin ese dolor se fue (Ana, Santa María, 2013).

Las emociones no se dan de manera individual. Surgen entre individuos y grupos de individuos. En sus escritos Le Breton (2012) nos dice que las emociones:

Son relaciones y por tanto son el producto de una construcción social y cultural y se expresan en un conjunto de signos que el hombre siempre tiene la posibilidad de desplegar, incluso si no las sienten. La emoción es a la vez interpretación, expresión, significación, relación, regulación de un intercambio; se modifica de acuerdo con el público, el contexto, se diferencia en su intensidad, e incluso en sus manifestaciones, de acuerdo a la singularidad de cada persona (Le Breton, 2012, p. 69).

Los sentimientos por tanto son relacionales (Bericat, 2012; Le Breton, 2012), se desarrollan de acuerdo con las relaciones que tenemos con otros. 
Para entender el caso de estos jóvenes, la definición de Besserer (2000) es muy útil. El autor realiza una articulación directa entre las formas de gobernar y las normas sentimentales, introduciendo la dimisión del poder en el estudio de las emociones. Él argumenta que cada forma de gobernar, según el contexto sociopolítico, establece reglas emocionales. En los casos que estoy presentando el gobierno americano impone sentimientos de miedo, de terror. Esto se puede ver claramente en los relatos de los jóvenes y cómo la creciente criminalización de los migrantes y el gobernar a través de la deportabilidad, haciéndolos sujetos "deportables" crea sentimientos de miedo, que influyen e impactan en sus vidas y las relaciones que van desarrollando a través de los años con los miembros de su familia, con sus compañeros en la escuela, sus maestros y con otros habitantes de la ciudad.

Miedo es lo que estos jóvenes, hombres y mujeres sienten en sus vidas cotidianas y se manifiesta de diversas maneras. El Estado impone un régimen de terror sobre aquellos que considera ilegales pero que aun así necesita para que su sistema continúe funcionando. Los jóvenes en los trabajos que lograban obtener se veían forzados a aceptar salarios más bajos, con menores prestaciones porque el no tener documentos que legalicen su estadía les impide reclamar contrataciones justas y luchar por sus derechos humanos, desencadenando sentimientos de desesperanza y miedo.

El entrar al DACA trajo a los jóvenes cambios en sus emociones. El tener cierta seguridad en su situación legal en los Estados Unidos les quitó el miedo que habían estado sintiendo y por unos años lograron sentir calma en sus vidas. Aurelio nos cuenta:

Yo me di cuenta que no tenía papeles cuando traté de conseguir un trabajo en un negocio y mi papá me dijo que para ese trabajo necesitaba papeles especiales, que yo no tenía. Todos aquí sabemos que hay gente indocumentada en los files, pero nunca pensé que yo era uno de ellos. En ese momento sentí como que una nube gris se posaba sobre mí. Todo lo que había oído sobre aquellos, ahora era yo. It was a strange feeling. I was sad. Pero sobre todo me dio miedo ... Con el DACA puedo conseguir el trabajo que quiera y caminar tranquilo por la calle (Aurelio, Santa María, 2016).

Otra emoción que los jóvenes frecuentemente mencionaban era la desilusión. La desilusión que sentían al terminar la preparatoria y darse cuenta lo difícil que su vida empezaba a ser y sería debido a que no tenían papeles. Y cómo esta sensación se fue desvaneciendo entre los jóvenes DACA. Alejandro comenta:

Al salir de la high school me sentí muy desilusionado conmigo, con la vida, con mi familia. I did not know what to do. En los files no quería trabajar, pero qué me quedaba. Entonces oí del DACA, todos tenían miedo, que no, que había que dar información de la familia, que era un truco, but, I did it. En el momento que recibí mis papeles sentí que una nube se levantó (Alejandro, Santa María, 2013).

Los jóvenes comentan que al recibir sus papeles sentían cómo la sombra bajo la que vivían se desvanecía y podían tener ilusiones una vez más, hacer planes, soñar.

El decidir hacer su aplicación para el DACA, para la mayoría de los entrevistados fue una decisión familiar. Por un lado, muchos necesitaron de la ayuda de sus padres para reunir la cantidad de dinero para pagar la cuota necesaria. Por otro lado, algunos miembros de las familias no estaban de acuerdo en que dieran la información de la familia a las autoridades por miedo a que esta después fuera utilizada por el gobierno para deportar a los miembros indocumentados, lo que generó que algunos jóvenes tardaran varios meses en tomar la decisión. Teresa nos platica: 
Cuando le platiqué a mi familia que quería aplicar al DACA, primero dijeron que sí, pero pronto mis hermanos dijeron que no. Ellos llegaron más grandes y no calificaban. Me dijeron que por mí sabrían de la familia y que la migra vendría por todos. Fueron discusiones muy dolorosas. Lloramos por tres meses. Me decían que para qué quería estudiar si era mujer y me iba a casar y dejar todo (Teresa, Santa María, 2014).

El caso de Teresa es representativo de la situación de muchas jóvenes que quieren estudiar, pero aún su familia piensa que por ser mujeres su futuro es casarse y tener hijos. En parte, los familiares quieren que estudien, que terminen la preparatoria, y también que lleguen al colegio estatal, pero cuando hay que tomar decisiones, estos argumentos siguen siendo utilizados para mantener a las mujeres en la casa. En el caso de Teresa, tardó tres meses, entre llantos y miedos, para convencer a su familia de que buscar su registro en DACA era lo correcto, no solo por sus estudios, también para su futuro en Estados Unidos y evitar una deportación.

La vida en las familias de estatus legal mixto es complicada, así como en algunas encontramos muestras de gran solidaridad, en otras hay tensiones y celos entre sus miembros, especialmente entre los hijos que tienen papeles y aquellos que no. Los que sí tienen, culpan a los que no por las limitantes que ocasionan en sus vidas, por ejemplo, el no poder viajar o tomar vacaciones familiares, ante esto los indocumentados se sienten con menos oportunidades, tienden a sentirse tristes y deprimidos por no tener las mismas oportunidades que sus hermanos (Barros, 2015b). Los jóvenes comentaron en sus entrevistas que estas emociones tendían a cambiar con el DACA, ya que no se sentían tan desprotegidos y sin oportunidades como antes. Gloria nos dice:

A los 15 años me quedé embarazada y tuve que dejar la escuela. No podía creer lo que me estaba pasando. Tuve a mi hijo sola, en casa de una tía, mi papá me corrió. Fue hasta que vio la bebé que me dejó regresar. Mi hermana terminó la High School y consiguió un buen trabajo y yo me fui al campo. La vida fue muy dura and my sister kept making me feel bad. Cuando nos dijeron del DACA me metí a clases, saqué mi título y saqué mi credencial. Me metí al college a estudiar criminal justice y ahora trabajo en un cooler con un mejor salario, me llevó mejor con mi familia, me siento mejor (Cata, Santa María 2016).

\section{Comentarios finales}

En este artículo hemos podido ver que aun en contextos con pocas oportunidades económicas como son los valles agrícolas, donde la gran mayoría de los empleos son para jornaleros migrantes, los jóvenes DACA lograron continuar sus estudios y la mayoría encontraron trabajo fuera del campo, en actividades mejor remuneradas y más atractivas para su desarrollo personal.

Es importante resaltar el interés que los jóvenes tienen por continuar estudiando, pues ellos saben que estos les darán mejores oportunidades en la vida; sin embargo, hay que señalar que las mujeres de nuestro estudio encontraron mayores retos que los hombres, tanto para continuar con sus carreras como para aplicar al DACA. Enfrentando dudas y cuestionamientos por parte de sus familias. La decisión de entrar al DACA fue difícil para todos, sin embargo, las mujeres fueron más cuestionadas por 
sus familiares. Aplicar al DACA trajo dudas y miedos a todos los miembros de la familia, quienes temían que sus datos fuesen utilizados por el gobierno para ser posteriormente deportados, pero una vez que recibieron la credencial y tuvieron el permiso para trabajar se pudieron ver cambios importantes en la vida de los jóvenes. El saber que ya no podían ser deportados y que tenían la capacidad de elegir, les trajo esperanza, les quitó o redujo el miedo en que vivían; los sacó de las sombras en que tuvieron que meterse y les permitió una vez más sentirse parte del país que siempre pensaron era su país y continuar construyendo sus sueños.

Sin embargo los tiempos de Trump han traído nuevas incertidumbres, aterrorizando a los indocumentados en Estados Unidos. Durante su campaña manifestó que una vez que fuera presidente cancelaría el programa DACA, lo cual el martes 5 de septiembre de 2017 anunció como un hecho, aun cuando dio seis meses para que el Congreso presente una solución para la vida de estos jóvenes, provocó una gran consternación entre la población migrante. Pero estos jóvenes tienen educación, conocen la vida política de Estados Unidos, muchos de ellos son activistas y están dispuestos a trabajar por su futuro.

A través de los ejemplos de vida de estos jóvenes hemos podido conocer un poco más la experiencia de vida de miles de jóvenes que llegaron de niños, crecieron en Estados Unidos, son bilingües y biculturales. Ellos decidieron que la educación es el mejor camino para superarse y apoyar a la sociedad en la que viven, por lo que esperamos que el Congreso y la sociedad en general valoren la importancia que tiene esta generación de mujeres y hombres para el futuro de su país y el daño que les haría, no solo a ellos sino a toda una generación de jóvenes que viven y conviven con estas familias y que tienen la ciudadanía americana.

\section{Referencias}

Aquino, A. (2015). "Porque si llamas al miedo, el miedo te friega": La ilegalización de los trabajadores migrantes y sus efectos en las subjetividades. Estudios Fronterizos, 16(32), 75-98.

Barros, M. (2009). La calle Guadalupe. En B. M. Valenzuela y M. Calleja (Comps.), Empresarios migrantes mexicanos en Estados Unidos (pp. 211-234). México: Universidad de Guadalajara.

Barros, M. (2015a). Apuntes sobre vivienda para migrantes y la crisis inmobiliaria en la California rural. Un estudio de caso. El Cotidiano, (191), 33-43.

Barros, M. (2015b). Jóvenes de origen mexicano en los remates del sur del Valle Central de California, E.U. Internacionales. Revista en Ciencias Sociales del Pacífico Mexicano, 1(1), 49-85.

Barros, M. (2017). "El Efecto Trump" en niños y jóvenes migrantes en la California Rural. Migración Ichan Tecolotl, 26(304).

Barros, M. y García, E. (2015). Jóvenes mixtecos migrantes de Oaxaca y el DACA. Estudios de caso en el valle de Santa María, California. Revista Con-temporánea, 2(4). Recuperado de http://con-temporanea.inah.gob.mx/node/111

Beneficiarán a más "Soñadores". (8 de agosto de 2012). El Universal. Recuperado de http://www.eluniversal.com.mx/internacional/78921.html 
Bericat, E. (2012). Emotions. Sociopedia. ISA. doi:10.1177/205684601361

Besserer, F. (2000). Sentimientos (in)apropiados de las mujeres migrantes: Hacia una nueva ciudadanía. En D. Barrera y C. Oehmichen (Coords.), Migración y relaciones de género en México (371-388). México: Grupo Interdisciplinario sobre Mujer, Trabajo y Pobreza, Universidad Nacional Autónoma de México, Instituto de Investigaciones Antropológicas.

Cornelius, W. A., FitzGerald, D., Lewin, F. P. y Muse-Orlinoff, L. (2010). Mexican Migration and the Us Economic Crisis. A Transnational Perspective. California, Estados Unidos de América: Center for Comparative Immigration Studies, University of California.

De Genova, N. (2002). Migrant "Illegality" and Deportability in Everyday Life. Annual Review of Anthropology, 31, 419-447.

Dinan, S. (14 de agosto de 2017). Dreamers face immigration showdown over amnesty with Trump, courts. The Washington Times. Recuperado de http://www.washingtontimes.com/news/2017/aug/14/daca-faces-challenges-trump-courts/

Dowling, J. A. e Inda, J. X. (Eds.) . (2013). Governing Immigration Through Crime. A Reader. California, Estados Unidos de América: Stanford California Press.

Fix, M. E. y Zimmermann, W. (2001). All Under One Roof: Mixed-Status Families in an Era of Reform, International Migration Review, 35(2), 397-419.

Hughes, E. (1960). The Profession in Society. The Canadian Journal of Economics and Political Science, XXVI(1), 54-61.

Kochhar, R. (2008). Latino Labor Report: Construction Reverses Growth for Latinos. Washington, Distrito de Columbia, Estados Unidos de América: Pew Hispanic Center.

Kochhar, R. (2009). Unemployment Rises Sharply Among Latino Immigrants in 2008. Washington, Distrito de Columbia, Estados Unidos de América: Pew Hispanic Center.

Le Breton, D. (2012). Por una antropología de las emociones. Revista Latinoamericana de Estudios sobre Cuerpos, Emociones y Sociedad, 4(10), 69-79. Recuperado de http://www.relaces.com.ar/index.php/relaces/article/view/208

Levine, D. y Cooke K. (14 de febrero de 2017). Mexican 'DREAMer'Nabbed in Immigration Crackdown. Recuperado de http://www.reuters.com/article/us-usa-trump-immigration-arrest-exclusiv/mexican-dreamer-nabbed-in-immigrant-crackdown-idUSKBN15T307

Mathema, S. (9 de enero de 2017). Ending DACA Will Cost States Billions of Dollars. Recuperado del sitio de Internet Center for American Progress: https://www.americanprogress.org/issues/immigration/news/2017/01/09/296125/ending-daca-will-cost-states-billions-of-dollars/

Merica, D. (16 de Agosto de 2017). Trump Says Both Sides to Blame Amid Charlottesville Blacklash. Recuperado de CNN politics: http://edition.cnn.com/2017/08/15/ politics/trump-charlottesville-delay/index.html

O`Neil, H. (25 de agosto de 2012). U. S. Immigration Policy Splits Families when Parents are Deported. The Denver Post. Recuperado de http://www.denverpost. com/nationworld/ci_21401857/u-s-immigration-policy-splits-families-when-parents\#ixzz24hrk0418

Suárez-Orozco, C., Suárez-Orozco, M. y Todorova, I. (2008). Learning a New Land. Immigrant Students in American Society. Massachussetts, Estados Unidos de América: The Belknap Press of Harvard University Press. 
Truax, E. (2013). Dreamers. La lucha de una generación por su sueño americano. México: Océano.

United States Census Bureau. (2010). QuickFacts, United States. Recuperado de https:// www.census.gov/quickfacts/fact/table/US/PSTo45216

United States Citizen and Immigration Services (USCIS). (2012). Consideration of Deferred Action for Childhood Arrivals (DACA). Recuperado de http://act.americasvoiceonline.org/page/m/327377f8/1fb6e4af/1e93d9fc/6f20de74/2111572833/ VEsH/

United States Citizen and Immigration Services (USCIS). (2015). Immigration and Citizenship Data. Recuperado de https://www.uscis.gov/tools/reports-studies/immigration-forms-data

Wong, T. K., García, A. D., Abrajano, M., FitsGerald, D., Ramakrishnan, K. y Le, S.(15 de septiembre de 2013). Undocumented No More. A Nationwide Analysis of Deferred Action for Chilhood Arrivals, or DACA. Recuperado de https://www.americanprogress.org/issues/immigration/reports/2013/09/20/74599/undocumented-no-more/

\section{Entrevistas}

Alejandro. (Julio de 2013). Entrevistado por la autora, Santa María, California, Estados Unidos de América.

Alfredo. (Julio de 2013). Entrevistado por la autora, Santa María, California, Estados Unidos de América.

Ana. (Junio de 2013). Entrevistada por la autora, Santa María, California, Estados Unidos de América.

Aurelio. (Octubre de 2016). Entrevistado por la autora, octubre, Santa María, California, Estados Unidos de América.

Carmen. (Octubre de 2014). Entrevistada por la autora, Santa María, Estados Unidos de América.

Carmen. (Febrero de 2015). Entrevistada por la autora, Santa Maria, California, Estados Unidos de América.

Cata. (Octubre de 2016). Entrevistada por la autora, Santa María, California, Estados Unidos de América.

Felicia. (Febrero de 2015). Entrevistada por la autora, Santa María, California, Estados Unidos de América.

Felipe. (Septiembre de 2012). Entrevistado por el autor, Santa María, California, Estados Unidos de América.

Gerardo. (Febrero de 2015). Entrevistado por la autora, Santa María, California, Estados Unidos de América.

Teresa. (Octubre de 2014). Entrevistado por la autora, Santa María, California, Estados Unidos de América. 


\begin{abstract}
Magdalena Barros Nock
Mexicana. Doctora en Estudios de Desarrollo, Instituto Internacional de Ciencias Sociales de la Universidad Erasmus de Rotterdam, Países Bajos. Profesora - investigadora de tiempo completo titular C en el Centro de Investigaciones y Estudios Superiores en Antropología Social (CIESAS) Ciudad de México. Líneas de investigación: migración internacional, familia de estatus legal mixto, jóvenes y niños migrantes; estudios de género; violencia; economía étnica. Entre sus publicaciones: Barros, M. y García, E. (2015). Jóvenes indígenas migrantes de Oaxaca y el DACA. Estudios de caso en el Valle de Santa María, California. Revista Con-temporánea, primera época, 2(4).
\end{abstract}

